\title{
A Simple but Efficient Approach for Testing Fuzzy Hypotheses
}

\author{
Abbas Parchami ${ }^{1}$, S. Mahmoud Taheri ${ }^{2 *}$, Bahram Sadeghpour Gildeh ${ }^{3}$ and Mashaallah Mashinchi ${ }^{1}$
}

\author{
*Correspondence: \\ sm_taheri@ut.ac.ir \\ ${ }^{2}$ Faculty of Engineering Science, \\ College of Engineering, University \\ of Tehran, Tehran, Iran \\ Full list of author information is \\ available at the end of the article
}

\begin{abstract}
In this paper, a new method is proposed for testing fuzzy hypotheses based on the following two generalized $p$-values: (1) the generalized $p$-value of null fuzzy hypothesis against alternative fuzzy hypothesis and (2) the generalized $p$-value of alternative fuzzy hypothesis against null fuzzy hypothesis. In the proposed method, each generalized $p$-value is formulated on the basis of Zadeh's probability measure of fuzzy events. The introduced $p$-value method has several advantages over the common $p$-value methods for testing fuzzy hypotheses. A few illustrative examples and also an agricultural example, based on a real-world data set, are given to clarify the proposed method.
\end{abstract}

Keywords: Fuzzy statistics, Fuzzy hypothesis, Testing hypothesis, $p$-value

\section{Introduction}

After the inception of the concept of fuzzy set by Zadeh [23], many statistitions have extended different methods of testing statistical hypotheses using the fuzzy set theory, e.g. see $[3,4,18,19]$. Beside the mentioned works on testing hypothesis in fuzzy environment, some works have been done by researchers based on the concept of $p$-value. Filzmoser and Viertl [8] worked on the problem of testing hypotheses and introduced a fuzzy $p$-value when the observations are fuzzy and hypotheses are crisp, also see [6, 21]. For instance, when the form of the rejection region is $T \geq t_{r}$, the $\delta$ cut of their proposed fuzzy $p$-value is

$$
\left[P_{\theta_{0}}\left(T \geq t_{2}(\delta)\right), P_{\theta_{0}}\left(T \geq t_{1}(\delta)\right)\right], \quad \delta \in(0,1],
$$

in which $\theta_{0}$ is the boundary of null hypothesis and $\tilde{t}_{\delta}=\left[t_{1}(\delta), t_{2}(\delta)\right]$ is the $\delta$ cut of the test statistic.

Using the extension principle, Parchami et al. [14] discussed on testing fuzzy hypotheses with crisp data and introduced the concept of the fuzzy $p$-value for such situations. For instance, when the form of the rejection region is $T \geq t_{r}$, the $\delta$ cut of their fuzzy $p$-value is

$$
\left[P_{\theta_{1}(\delta)}(T \geq t), P_{\theta_{2}(\delta)}(T \geq t)\right], \quad \delta \in(0,1],
$$

in which $\left(H_{0 b}\right)_{\delta}=\left[\theta_{1}(\delta), \theta_{2}(\delta)\right]$ is the $\delta$ cut of the null hypothesis boundary and $t$ is the observed test statistic.

Parchami et al. [15] and Fazlalipor et al. [7] combined the two abovementioned ideas of Filzmoser and Viertl [8] and Parchami et al. [14] by two different approaches which lead to the same solution for testing fuzzy hypotheses based on fuzzy data. For instance, when the form of the rejection region is $T \geq t_{r}$, the $\delta$ cut of their proposed fuzzy $p$-value is

(c) 2016 Parchami et al. Open Access This article is distributed under the terms of the Creative Commons Attribution 4.0 International License (http://creativecommons.org/licenses/by/4.0/), which permits unrestricted use, distribution, and reproduction in any medium, provided you give appropriate credit to the original author(s) and the source, provide a link to the Creative Commons license, and indicate if changes were made. 


$$
\left[P_{\theta_{1}(\delta)}\left(T \geq t_{2}(\delta)\right), P_{\theta_{2}(\delta)}\left(T \geq t_{1}(\delta)\right)\right], \delta \in(0,1]
$$

in which $\left(H_{0 b}\right)_{\delta}=\left[\theta_{1}(\delta), \theta_{2}(\delta)\right]$ and $\tilde{t}_{\delta}=\left[t_{1}(\delta), t_{2}(\delta)\right]$ are $\delta$ cuts of null hypothesis boundary and test statistic, respectively.

Also, Geyer and Meeden [9] investigated the concepts of fuzzy $p$-value and fuzzy confidence interval when both the hypotheses and data are crisp.

Unlike these studies, another efficient and simple $p$-value-based method for testing fuzzy hypotheses is presented in this paper. The proposed method is on the basis of the probability measure of fuzzy event introduced by Zadeh [22]. Also, it must be mentioned that all results of this study coincide with the results of testing classical hypotheses, when the hypotheses reduce to two crisp sets on the parameter space.

This paper is organized as follows. Some preliminaries, motivations and basic definitions about testing fuzzy hypotheses are reviewed in Section "Fuzzy Hypotheses: Motivation and Basic Definitions". In Section "Testing Fuzzy Hypotheses Based on a New $p$-Value-Based Approach", we present a new $p$-value-based approach for testing fuzzy hypotheses. Some illustrative examples are given in Section "Illustrative Examples". An agricultural applied example is presented in Section "Application to Agricultural Studies". Also, a conclusion is given in the final section.

\section{Fuzzy Hypotheses: Motivation and Basic Definitions}

Here, we are going to briefly review some basic concepts which are needed or developed through this paper.

\section{Testing Statistical Hypotheses}

Let $\mathbf{X}=\left(X_{1}, \ldots, X_{n}\right)$ be a random sample with the observed value $\mathbf{x}=\left(x_{1}, \ldots, x_{n}\right)$, where $X_{i}$ has the probability density function (p.d.f.) or the probability mass function (p.m.f.) $f\left(x_{i} ; \theta\right), i=1, \ldots, n$, with the unknown parameter $\theta \in \Theta \subseteq \mathbb{R}$. It will be assumed that the functional form of $f(x ; \theta)$ is known. The problem of testing statistical hypotheses is to decide whether to accept (or reject) the null hypothesis " $H_{0}: \theta \in \Theta_{0} \subset \Theta$ " against " $H_{1}: \theta \in \Theta_{0}^{c}=\Theta-\Theta_{0}$ ", based on the random sample $\mathbf{X}$. Usually, statistical hypotheses are one of the following forms:
(i) $H_{0}: \theta=\theta_{0}$ versus $H_{1}: \theta=\theta_{1}\left(\theta_{0}>\theta_{1}\right)$
(ii) $H_{0}: \theta=\theta_{0}$ versus $H_{1}: \theta=\theta_{1} \quad\left(\theta_{0}<\theta_{1}\right)$
(iii) $H_{0}: \theta \geq \theta_{0}$ versus $H_{1}: \theta<\theta_{0}$
(iv) $H_{0}: \theta \leq \theta_{0}$ versus $H_{1}: \theta>\theta_{0}$
(v) $H_{0}: \theta=\theta_{0}$ versus $H_{1}: \theta \neq \theta_{0}$

In which $\theta_{0}$ and $\theta_{1}$ are two known numbers and we named them the boundary of the null and alternative hypotheses, respectively. A test $\phi$ is said to be a test of (significance) level $\alpha \in[0,1]$ if $\alpha_{\phi} \leq \alpha$, where $\alpha_{\phi}=\sup _{\theta \in \Theta_{0}} P_{\theta}$ (rejection of $H_{0}$ ). Commonly, the statistical tests are based on a so-called test statistic $T(\mathbf{X})$. In a nonrandomized test, the space of possible values of the test statistic $T$ is decomposed into a rejection region and its complement, the acceptance region. Under some certain conditions, the rejection region usually takes one of the following forms:
(a) $T \leq t_{l}$
(b) $T \geq t_{r}$
(c) $T \notin\left(t_{1}, t_{2}\right)$, 
where $t_{l}, t_{r}$, or $t_{1}$ and $t_{2}$ are certain quantiles of the distribution of $T$, so that $\alpha_{\phi}=\alpha$. In case (c), we may obtain $t_{1}$ and $t_{2}$ by the equal tails method, so that $P_{\theta}\left(T \leq t_{1}\right)=P_{\theta}(T \geq$ $\left.t_{2}\right)=\alpha / 2$. The hypothesis $H_{0}$ is rejected if the value of $t=t(\mathbf{x})$ falls into the rejection region. In usual tests, the critical regions of testing hypotheses $(i)$ and (iii) are of form (1.a), the critical region of testing hypotheses (ii) and (iv) are of form (1.b) and the critical region of testing hypothesis $(v)$ is of form (1.c). For more details, see $[8,11,15]$.

\section{Fuzzy Hypotheses: Motivation}

The motivation of introducing fuzzy hypotheses can be briefly presented by considering the following example. Suppose that an experimenter is interested in evaluating the mean growth of a plant by measuring its diameter which is distributed by a normal distribution with unknown mean $\mu$ and known standard deviation $\sigma$. The traditional method is testing hypothesis " $H_{0}: \mu=\mu_{0}$ " against " $H_{1}: \mu \neq \mu_{0}$ ", for a certain value $\mu_{0}$, on the basis of a random sample $\mathbf{X}$, where $X_{i} \sim N\left(\mu, \sigma^{2}\right), i=1, \ldots, n$. But obviously, if the mean of the given sample is slightly different from $\mu_{0}$, then the null hypothesis is acceptable, and a considerable difference from $\mu_{0}$ makes $H_{0}$ unacceptable. Therefore, it is more appropriate and more reasonable to formulate the hypotheses $H_{0}$ and $H_{1}$ by fuzzy terms "near to $\mu_{0}$ " and "away from $\mu_{0}$ ", respectively. In other words, more realistic hypotheses are

$$
\left\{\begin{array}{l}
\tilde{H}_{0}: \mu \text { is near to } \mu_{0}, \\
\tilde{H}_{1}: \mu \text { is away from } \mu_{0},
\end{array}\right.
$$

which can be formulated by using Definition 1 . Having faced by such real situations leads statisticians to reformulate some classical and crisp hypotheses by using fuzzy hypotheses. Considering the above discussion, the main problem studied in this work is to test fuzzy hypotheses

$$
\left\{\begin{array}{l}
\tilde{H}_{0}: \theta \text { is } H_{0}(\theta), \\
\tilde{H}_{1}: \theta \text { is } H_{1}(\theta),
\end{array}\right.
$$

based on a random sample from a p.d.f. or p.m.f. $f(x ; \theta), \theta \in \Theta$ (for more details, see Definition 1). This problem is called the problem of testing fuzzy hypotheses. In this paper, motivated by [22], we propose a new $p$-value-based approach to such a problem. Concerning the common $p$-value-based methods, the introduced method has some important advantages. The main advantage of the introduced method is that it is based on both null and alternative hypotheses, while the common methods are based on null hypothesis only.

\section{Fuzzy Hypothesis and Its Boundary}

Here, we review some basic concepts about fuzzy hypotheses from Taheri and Behboodian [18] and Parchami et al. [15], which are used in Section "Testing Fuzzy Hypotheses Based on a New $p$-Value-Based Approach".

Definition 1. Any hypothesis of form " $\tilde{H}: \theta$ is $H(\theta)$ " is called a fuzzy hypothesis, where " $\theta$ is $H(\theta)$ " implies that $\theta$ is in a fuzzy set of $\Theta$, the parameter space, with membership function $H(\theta)$.

Note that the ordinary hypothesis " $H: \theta=\theta_{0}$ " is a fuzzy hypothesis with the membership function $H(\theta)=1$ at $\theta=\theta_{0}$, and zero otherwise, i.e. the indicator function of the crisp set $\left\{\theta_{0}\right\}$. 
Definition 2. (See also [1, 2]) (a) Fuzzy hypothesis $\tilde{H}: \theta$ is $H(\theta)$ is called a fuzzy one-sided hypothesis, if there exists $\theta_{1} \in \Theta$ so that: $(i) H(\theta)=1$ for $\theta \leq \theta_{1}\left(\theta \geq \theta_{1}\right)$ and (ii) $H$ is an decreasing (increasing) function of $\theta$ for $\theta>\theta_{1}\left(\theta<\theta_{1}\right)$.

(b) Fuzzy hypothesis $\tilde{H}: \theta$ is $H(\theta)$ is called a fuzzy two-sided hypothesis, if there exists an interval $\left[\theta_{1}, \theta_{2}\right] \subset \Theta$ so that: $(i) H(\theta)=1$ for $\theta \in\left[\theta_{1}, \theta_{2}\right]$ and (ii) $H$ is an increasing function of $\theta$ for $\theta \leq \theta_{1}$ and is a decreasing function for $\theta \geq \theta_{2}$.

Definition 3. The boundary of the fuzzy hypothesis $\tilde{H}$ is a fuzzy subset of $\Theta$ with membership function $H_{b}$ defined as follows:

(i) $H_{b}(\theta)=\left\{\begin{array}{ccc}H(\theta) & \text { for } & \theta \leq \theta_{1}, \\ 0 & \text { for } & \theta>\theta_{1}\end{array}\right\}$, if $\tilde{H}$ is one-sided and $H$ is increasing,

(ii) $H_{b}(\theta)=\left\{\begin{array}{ccc}H(\theta) & \text { for } & \theta \geq \theta_{1}, \\ 0 & \text { for } & \theta<\theta_{1}\end{array}\right\}$, if $\tilde{H}$ is one-sided and $H$ is decreasing,

(iii) $H_{b}(\theta)=H(\theta)$, if $\tilde{H}$ is two-sided.

Example 1. Let $X$ be an exponential random variable with unknown mean $\lambda$, i.e. $f(x ; \lambda)=\frac{1}{\lambda} e^{-\frac{x}{\lambda}}, x>0, \lambda>0$. Suppose that

$$
H(\lambda)=\left\{\begin{array}{lll}
e^{-(\lambda-3)^{2}} & \text { if } & \lambda \leq 3 \\
1 & \text { if } & \lambda>3
\end{array}\right.
$$

Then, the hypothesis " $\tilde{H}: \lambda$ is $H(\lambda)$ " is a fuzzy one-sided hypothesis. So, by Definition 3,

$$
H_{b}(\lambda)= \begin{cases}e^{-(\lambda-3)^{2}} & \text { if } \lambda \leq 3 \\ 0 & \text { if } \lambda>3\end{cases}
$$

is the boundary of the fuzzy hypothesis $\tilde{H}$ (see Fig. 1).

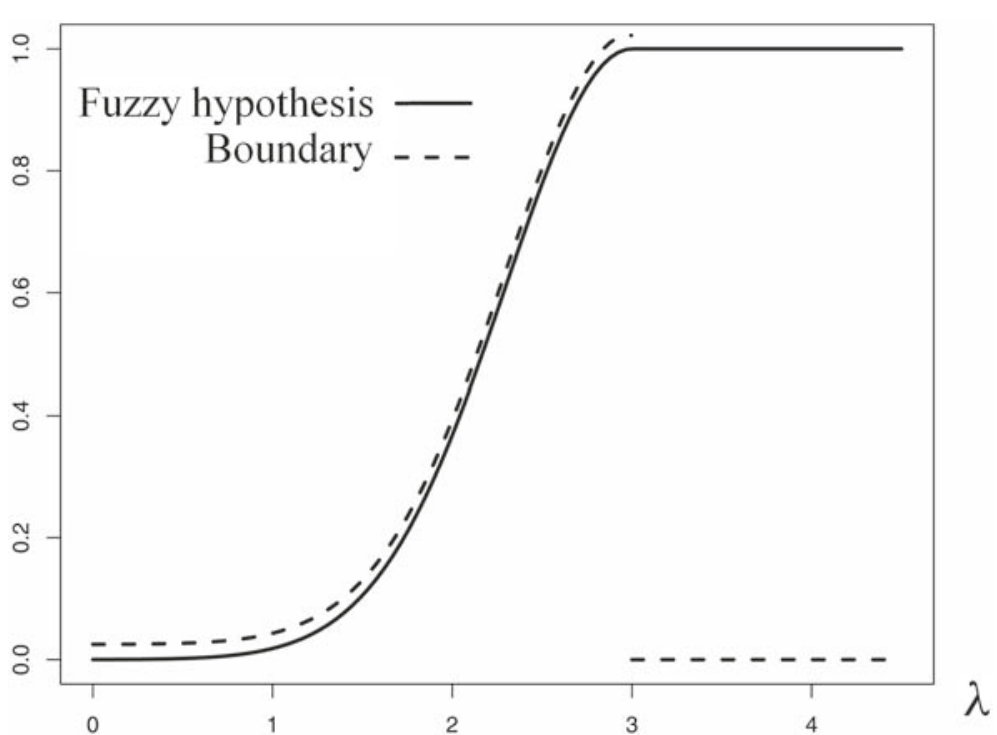

Fig. 1 The membership functions of the fuzzy hypothesis and its boundary in Example 1 


\section{Probability Measure Under a Fuzzy Hypothesis}

Definition 4. (Torabi and Behboodian [20]) Let the random variable $X$ have p.d.f. or p.m.f. $f(x ; \theta)$ and $\tilde{H}: \theta$ is $H(\theta)$ is a fuzzy hypothesis where $\int_{\theta} H(\theta) d \theta<\infty$. The weighted probability density function of $X$, under the fuzzy hypothesis $\tilde{H}$, is defined by

$$
f(x ; \tilde{H})=\int_{\theta} H^{*}(\theta) f(x ; \theta) d \theta,
$$

where $H^{*}(\theta)=\frac{H(\theta)}{\int_{\theta} H(\theta) d \theta}$ is the normalized membership function of $H(\theta)$. Replace integration by summation in discrete cases.

Remark 1. (Torabi and Behboodian [20]) (a) The normalized membership function is not necessarily a membership function, i.e. it may be greater than 1 for some values of $\theta$.

(b) Note that $f(x ; \tilde{H})$ in Definition 4 is a p.d.f., since $f(x ; \tilde{H})$ is nonnegative and $\int_{x} f(x ; \tilde{H}) d x=1$.

(c) If $H$ is the crisp hypothesis $H: \theta=\theta_{0}$, then $f(x ; \tilde{H})=f\left(x ; \theta_{0}\right)$.

Example 2. Let $X$ be a normal random variable with unknown mean $\mu$ and variance 0.7 . The weighted p.d.f. of $X$ under fuzzy hypothesis " $\tilde{H}: \mu \cong 4$ " is as follows:

$$
\begin{aligned}
f(x ; \mu \cong 4) & =\int_{\mu} H^{*}(\mu) f(x ; \mu) d \mu \\
& =\frac{2}{3} \int_{\mu} H(\mu)\left(\frac{1}{\sqrt{1.4 \pi}} e^{-\frac{(x-\mu)^{2}}{1.4}}\right) d \mu, x \in R,
\end{aligned}
$$

where $\mu \cong 4$ is defined by

$$
H(\mu)=\left\{\begin{array}{llr}
\frac{\mu-2}{2} & \text { if } & 2<\mu \leq 4 \\
2(4.5-\mu) & \text { if } & 4<\mu \leq 4.5 \\
0 & & \text { otherwise. }
\end{array}\right.
$$

The calculation process for the weighted p.d.f. of $X$ under fuzzy hypothesis $\mu \cong 4$ is drawn in Fig. 2 on the basis of Definition 4.

The major advantage of Definition 4 is that the weighted p.d.f. can integrate all possible p.d.f.s with different weights. The value of $H^{*}(\theta)$ can be understood as the weight of $f(x ; \theta)$, and the weighted p.d.f. can let different possible $f(x ; \theta)$ s play different roles in this integration (e.g. see Fig. 2 in Example 2).

\section{Testing Fuzzy Hypotheses Based on a New p-Value-Based Approach}

\section{The $p$-Value Approach}

Similar to the usual kinds of hypotheses which are listed at the first of Section "Fuzzy Hypotheses: Motivation and Basic Definitions", the fuzzy hypotheses can be usually modelled by one of the following forms:

(i) $\left\{\begin{array}{l}\tilde{H}_{0}: \theta \text { is approximately } \theta_{0}, \\ \tilde{H}_{1}: \theta \text { is approximately } \theta_{1},\end{array}\right.$ where $\operatorname{Def}\left(H_{0}\right)>\operatorname{Def}\left(H_{1}\right)$,

(ii) $\left\{\begin{array}{l}\tilde{H}_{0}: \theta \text { is approximately } \theta_{0}, \\ \tilde{H}_{1}: \theta \text { is approximately } \theta_{1},\end{array}\right.$ where $\operatorname{Def}\left(H_{0}\right)<\operatorname{Def}\left(H_{1}\right)$,

(iii) $\left\{\begin{array}{l}\tilde{H}_{0}: \theta \text { is approximately bigger than } \theta_{0}, \\ \tilde{H}_{1}: \theta \text { is approximately smaller than } \theta_{0},\end{array}\right.$ 


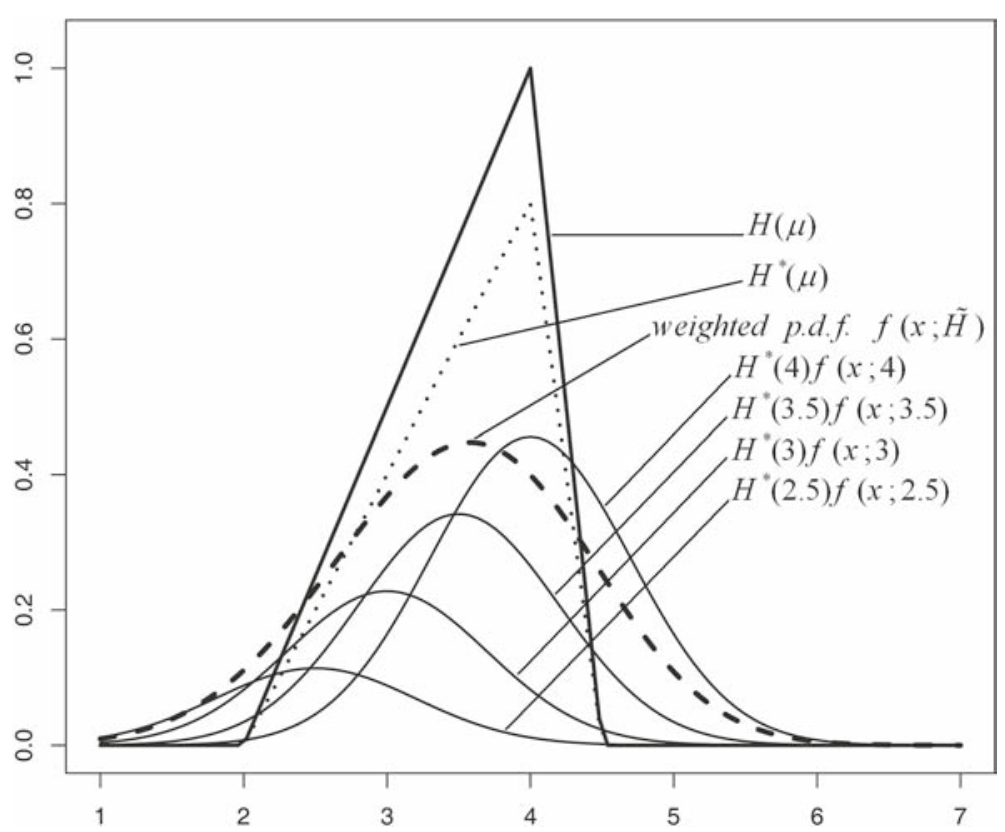

Fig. 2 Calculating normal weighted p.d.f. of $X$ under fuzzy hypothesis $\mu \cong 4$ in Example 2

(iv) $\left\{\begin{array}{l}\tilde{H}_{0}: \theta \text { is approximately smaller than } \theta_{0}, \\ \tilde{H}_{1}: \theta \text { is approximately bigger than } \theta_{0}\end{array}\right.$

(v) $\left\{\begin{array}{l}\tilde{H}_{0}: \theta \text { is near to } \theta_{0}, \\ \tilde{H}_{1}: \theta \text { is away from } \theta_{0},\end{array}\right.$

where $\theta_{0}$ and $\theta_{1}$ are two known numbers and $\operatorname{Def}($.$) is a defuzzifier function. It is obvious$ that critical regions of testing fuzzy hypotheses are similar to the critical regions of testing precise hypotheses which are formulated by Relations (1). In other words, the critical regions of testing fuzzy hypotheses (i) and (iii) are of form (1.a), the critical regions of testing fuzzy hypotheses (ii) and (iv) are of form (1.b) and the critical region of testing fuzzy hypothesis $(v)$ is of form (1.c). It must be noted that the critical regions of testing fuzzy hypotheses $(i)$ and $(i i)$ must be determined after defuzzification of the hypotheses, and it depends on the defuzzifier function.

Considering Definitions 1 and 3 , one can assert that " $\tilde{H}_{b}: \theta$ is $H_{b}(\theta)$ " is a fuzzy hypothesis, and therefore we can generalize the classical $p$-value for testing fuzzy hypothesis $\tilde{H}_{0}$ against $\tilde{H}_{1}$ as follows (see Subsection "Probability Measure Under a Fuzzy Hypothesis", for more details about the probability measure under a fuzzy hypothesis).

Definition 5. In testing fuzzy hypotheses problem, for any critical region of forms (1.a), (1.b) and (1.c), the $p$-value is respectively defined as

(a) $p$-value $=P_{\tilde{H}_{0 b}}(T \leq t)$

$$
=\int_{\theta} H_{0 b}^{*}(\theta) P_{\theta}(T \leq t) d \theta
$$

(b) $p$-value $=P_{\tilde{H}_{0 b}}(T \geq t)$

$$
=\int_{\theta} H_{0 b}^{*}(\theta) P_{\theta}(T \geq t) d \theta
$$


and

$$
\begin{aligned}
& \text { (c) } p \text {-value }= \begin{cases}2 P_{\tilde{H}_{0 b}}(T \geq t) & \text { if } t \geq m_{H_{0 b}} \\
2 P_{\tilde{H}_{0 b}}(T \leq t) & \text { if } t \leq m_{H_{0 b}}\end{cases} \\
& = \begin{cases}2 \int_{\theta} H_{0 b}^{*}(\theta) P_{\theta}(T \geq t) d \theta & \text { if } t \geq m_{H_{0 b}}, \\
2 \int_{\theta} H_{0 b}^{*}(\theta) P_{\theta}(T \leq t) d \theta & \text { if } t \leq m_{H_{0 b}},\end{cases}
\end{aligned}
$$

where $H_{0 b}^{*}(\theta)=\frac{H_{0 b}(\theta)}{\int_{\theta} H_{0 b}(\theta) d \theta}$ is the normalized membership function of the boundary in the fuzzy null hypothesis, $t$ is the observed value of test statistic $(T)$ and $m_{H_{0 b}}$ is the median of the weighted distribution of $T(\mathbf{X})$ under the boundary of the fuzzy null hypothesis $\tilde{H}_{0 b}$. Replacement of integration by summation is needed in discrete case.

Remark 2. In contrast with previous $p$-value methods in fuzzy environments (reviewed in Section "Introduction"), the proposed $p$-value in this study is a real number on unit interval which is formulated according to the probability measure under fuzzy hypothesis.

Remark 3. When the hypotheses are crisp rather than fuzzy, the membership function of the fuzzy boundary is reduced to the indicator function of a single point, i.e. the indicator function of the boundary $\theta_{0}$. Then, the introduced $p$-value for cases (a), (b) and (c) in Definition 5 are reduced to classical $p$ values
(a) $P_{\theta_{0}}(T \leq t)$,
(b) $P_{\theta_{0}}(T \geq t)$

and

(c) $2 \min \left\{P_{\theta_{0}}(T \leq t), P_{\theta_{0}}(T \geq t)\right\}=\left\{\begin{array}{ll}2 P_{\theta_{0}}(T \geq t) & \text { if } t \geq m_{\theta_{0}} \\ 2 P_{\theta_{0}}(T \leq t) & \text { if } t \leq m_{\theta_{0}}\end{array}\right.$,

where $\theta_{0}$ is the boundary of the null hypothesis and $m_{\theta_{0}}$ is the median of $T$ under $\theta_{0}$, see [8] and page 381 of [11].

\section{Decision Rule}

In testing fuzzy hypothesis $\tilde{H}_{0}$ against $\tilde{H}_{1}$, suppose that $p_{01}$ is the $p$-value in testing $\tilde{H}_{0}$ against $\tilde{H}_{1}$ and $p_{10}$ is the $p$-value in testing $\tilde{H}_{1}$ versus $\tilde{H}_{0}$. Now we are going to extend the proposed method of Emadi and Arghami [5] for testing fuzzy hypotheses. It must be mentioned that the proposed decision rule omit several weaknesses of the classical $p$-value-based tests which are point out in the next subsection.

Decision rule: Accept $\tilde{H}_{1}$ with confidence factor $\frac{p_{10}}{p_{01}+p_{10}}$ if $p_{01}<p_{10}$; otherwise, accept $\tilde{H}_{0}$ with confidence factor $\frac{p_{01}}{p_{01}+p_{10}}$. Therefore in this method, the "confidence factor into the given decision" can be introduced by

$$
\mathrm{CF}=\frac{p_{01}}{p_{01}+p_{10}} I\left(p_{01} \geq p_{10}\right)+\frac{p_{10}}{p_{01}+p_{10}} I\left(p_{01}<p_{10}\right)
$$

in which $I($.) is the indicator function.

\section{Advantages of the Proposed Method}

Some advantages of the proposed decision rule with respect to the decision rule of the classical $p$-value-based tests (i.e. reject null hypothesis at significance level $\alpha$ iff $\alpha>p_{01}$ ) are presented as follows: 
1. The proposed decision rule is a function of both fuzzy hypotheses $\tilde{H}_{0}$ and $\tilde{H}_{1}$, while the current fuzzy $p$-value methods are based on null fuzzy hypothesis (e.g. see Remark 7 in [14]).

2. The proposed decision rule is symmetric with respect to the hypotheses. In other words, the acceptance (rejection) $\tilde{H}_{0}$ versus $\tilde{H}_{1}$ in this method is equivalent to the rejection (acceptance) $\tilde{H}_{1}$ versus $\tilde{H}_{0}$, while the previous $p$-value-based methods in fuzzy environments do not have such reasonable property (see [16] for investigation on misleading statistical evidence by the current $p$-value methods). Also, there exist such a symmetry in the introduced confidence factor, since $\operatorname{CF}\left(\tilde{H}_{0}\right)=1-\operatorname{CF}\left(\tilde{H}_{1}\right)$.

3. When $\tilde{H}_{1} \longrightarrow \tilde{H}_{0}$, then $p_{10} \longrightarrow p_{01}$ and therefore, $\mathrm{CF} \longrightarrow \frac{1}{2}$ which indicates a similar supporting data from both hypotheses. It must be mentioned that not only the usual $p$-value methods for testing crisp hypotheses do not have this property but also the fuzzy $p$-value-based methods for fuzzy environments do not have such property, e.g. see $[8,17]$.

4. The previous fuzzy $p$-value-based methods may not lead the user to a clear decision in two-sided tests, when the fuzziness of the data or/and the fuzziness of the null hypothesis is/are high (for more details, see $[7,8,14,15])$. This problem is solved in the proposed approach by omitting no-decision area in Formula (4).

\section{Illustrative Examples}

Example 3. Let $x=2.25$ be an observation from $N(\mu, 1)$ distribution. We wish to test $\left\{\begin{array}{l}\tilde{H}_{0}: \mu \text { is approximately smaller than } 1.5, \\ \tilde{H}_{1}: \mu \text { is approximately bigger than } 1.5,\end{array}\right.$

where $\tilde{H}_{0}$ and $\tilde{H}_{1}$ have membership functions

$$
H_{0}(\mu)=\left\{\begin{array}{llr}
1 & \text { if } & \mu \leq 0 \\
\frac{3-\mu}{3} & \text { if } & 0<\mu \leq 3 \\
0 & \text { if } & \mu \geq 3
\end{array}\right.
$$

and $H_{1}(\mu)=1-H_{0}(\mu)$ (see Fig. 3). Considering Definition 3, the membership function of the boundary of fuzzy null hypothesis is

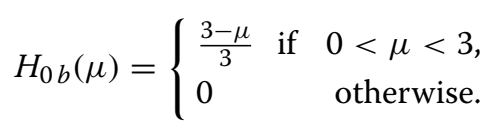

The fuzzy hypotheses in this example can be modelled by form (iv) and so the rejection region is of form (1.b). Therefore, considering Relation (3), one can compute $p$-value based on the weighted p.d.f. of $X$ under $\tilde{H}_{0 b}$ as follows:

$$
\begin{aligned}
p_{01} & =P_{\tilde{H}_{0 b}}(X \geq 2.25) \\
& =\int_{\mu} H_{0 b}^{*}(\mu) P_{\mu}(X \geq 2.25) d \mu \\
& =\int_{0}^{3} \frac{(3-\mu) / 3}{1.5}[1-\Phi(2.25-\mu)] d \mu \\
& =0.156,
\end{aligned}
$$

which is equivalent to the area of dark grey surface in Fig. 3. To decide on the basis of the proposed decision rule, we need to calculate the $p$-value of testing $\tilde{H}_{1}$ versus $\tilde{H}_{0}$. Hence, one can similarly compute $p_{10}$ as follows, which is equivalent to the area of light grey surface in Fig. 3 


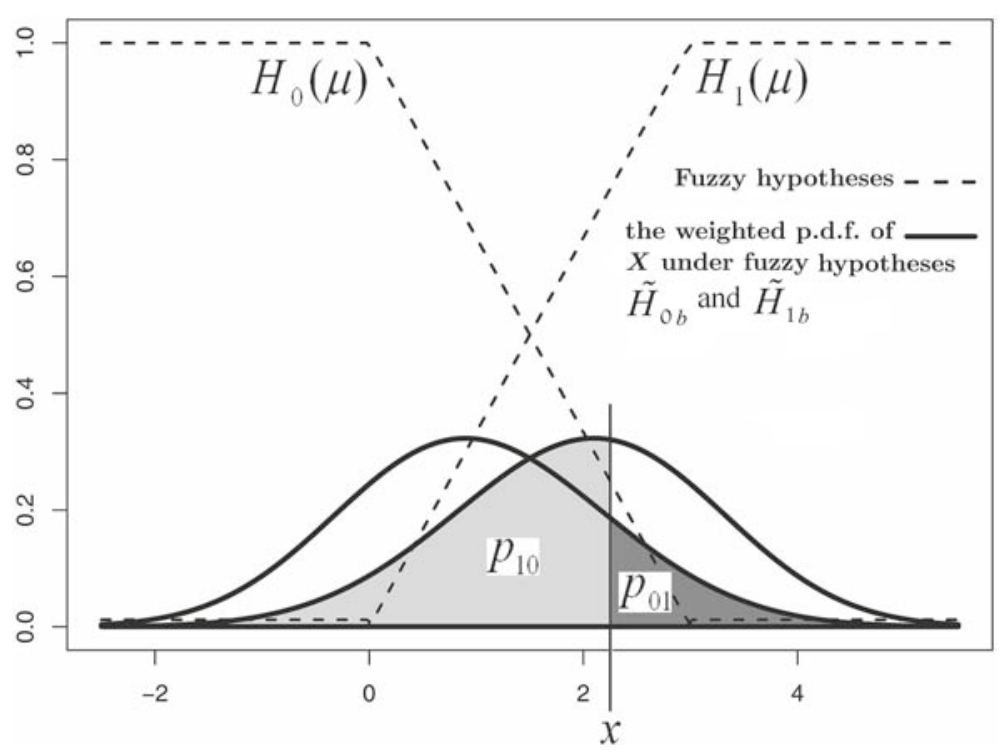

Fig. $3 p_{01}$ and $p_{10}$ in Example 3 based on the weighted p.d.f. of the test statistic under the boundary of fuzzy hypotheses

$$
\begin{aligned}
p_{10} & =P_{\tilde{H}_{1 b}}(X \leq 2.25) \\
& =\int_{0}^{3} \frac{1-[(3-\mu) / 3]}{1.5} \Phi(2.25-\mu) d \mu \\
& =0.572 .
\end{aligned}
$$

Therefore, $p_{01}<p_{10}$, and so we accept $\tilde{H}_{1}$ against $\tilde{H}_{0}$ with confidence factor $\mathrm{CF}=$ $\frac{0.572}{0.156+0.572}=0.786$. Note that on the basis of the classical $p$-value method, one accepts $\tilde{H}_{0}$ against $\tilde{H}_{1}$ at any significance level $\alpha<p_{01}=0.156$. Although in this example, the result of the proposed method is in conflict with the result of the classical significance tests (e.g. at level 0.05), but we assert that the proposed method is much better according to the comparison of two grey surfaces in Fig. 3.

Example 4. Let $x=1.75$ be an observation from $N(\mu, 1)$ distribution. We wish to test

$$
\left\{\begin{array}{l}
\tilde{H}_{0}: \mu \text { is approximately } 4, \\
\tilde{H}_{1}: \mu \text { is approximately } 1,
\end{array}\right.
$$

where $\tilde{H}_{0}$ and $\tilde{H}_{1}$ have membership functions (see the first graph in Fig. 4)

$$
H_{0}(\mu)=\left\{\begin{array}{lll}
\mu-3 & \text { if } & 3<\mu \leq 4 \\
5-\mu & \text { if } & 4<\mu \leq 5 \\
0 & & \text { otherwise }
\end{array}\right.
$$

and

$$
H_{1}(\mu)=\left\{\begin{array}{lll}
\mu & \text { if } & 0<\mu \leq 1 \\
2-\mu & \text { if } & 1<\mu \leq 2 \\
0 & \text { otherwise }
\end{array}\right.
$$

Considering Definition 3, the membership function of the boundary of fuzzy null hypothesis is equal to the membership function of the fuzzy null hypothesis. Also, the above fuzzy hypotheses can be modelled by form (ii), and so the rejection region for 

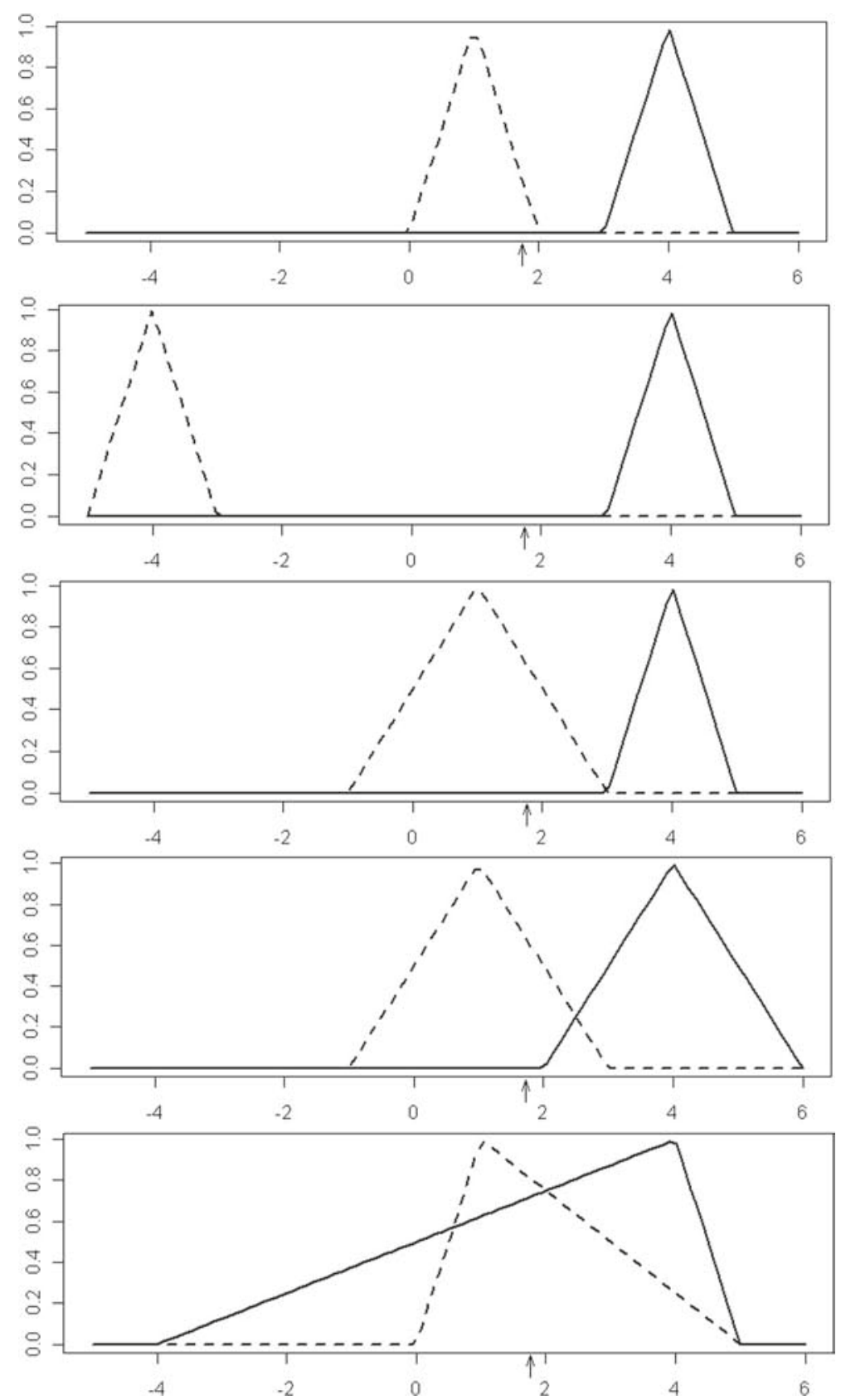

Fig. 4 The membership functions of fuzzy hypotheses $\tilde{H}_{0}$ and $\tilde{H}_{1}$ for five tests in Example 4 which are respectively drawn with a straight line and broken line

testing $\tilde{H}_{0}$ versus $\tilde{H}_{1}$ is of form (1.a). Therefore, considering Relation (2), one can compute $p$-value based on the weighted p.d.f. of $X$ under $\tilde{H}_{0 b}$ as follows:

$$
\begin{aligned}
p_{01} & =P_{\tilde{H}_{0 b}}(X \leq 1.75) \\
& =\int_{\mu} H_{0 b}^{*}(\mu) P_{\mu}(X \leq 1.75) d \mu \\
& =\int_{3}^{4}(\mu-3) \Phi(1.75-\mu) d \mu+\int_{4}^{5}(5-\mu) \Phi(1.75-\mu) d \mu \\
& =0.019 .
\end{aligned}
$$


To decide on the basis of the proposed decision rule, we need to calculate the $p$-value of testing $\tilde{H}_{1}$ versus $\tilde{H}_{0}$. Hence, one can similarly compute $p_{10}=P_{\tilde{H}_{1 b}}(X \geq 1.75)=$ 0.244 . Therefore, $p_{01}<p_{10}$, and so we accept $\tilde{H}_{1}$ against $\tilde{H}_{0}$ with confidence factor $\mathrm{CF}=$ $\frac{0.244}{0.019+0.244}=0.927$ (see the result of test 1 in Table 1 ).

To compare the results of several testing fuzzy hypotheses based on the proposed approach, five different tests are considered in this example by changing the fuzzy hypotheses (see Fig. 4). The membership functions of fuzzy hypotheses and the results of tests are presented in Table 1. Comparing the result of test 1 with the result of test 2 shows the sensitivity of the proposed approach to the location of the alternative fuzzy hypothesis (compare the first graph with the second one in Fig. 4). Also, comparing the result of test 1 with the result of test 3 shows the sensitivity of the proposed approach to the fuzziness of $\tilde{H}_{1}$. Instead of the current fuzzy $p$-value methods, the results of tests $1-5$ show that the proposed decision rule in this paper is a function of both fuzzy hypotheses $\tilde{H}_{0}$ and $\tilde{H}_{1}$ (see the first advantage from Subsection "Advantages of the Proposed Method").

Regarding to the used defuzzifier function $\operatorname{Def}(H)=\frac{\int_{\theta} \theta H(\theta) d \theta}{\int_{\theta} H(\theta) d \theta}$ in this example, the form of critical region is (1.b) for calculating $p_{01}$ in test 5 (since $\operatorname{Def}\left(H_{0}\right)<\operatorname{Def}\left(H_{1}\right)$ ), while in tests $1-4$, the form of critical region is of form (1.a).

Example 5. The lifetime $X$ of lamps (in terms of hour) produced by a factory is normally distributed with unknown mean $\mu$ and standard deviation $\sigma=120$. In a random sample of size $n=36$ lamps, we observe $\bar{x}=1327 \mathrm{~h}$. We wish to test

$$
\left\{\begin{array}{l}
\tilde{H}_{0}: \mu \text { is approximately } 1300, \\
\tilde{H}_{1}: \mu \text { is approximately } 1500
\end{array}\right.
$$

where their membership functions are (see Fig. 5)

$$
H_{0}(\mu)=\left\{\begin{array}{llr}
\frac{\mu-1200}{100} & \text { if } & 1200<\mu \leq 1300 \\
\frac{1400-\mu}{100} & \text { if } & 1300<\mu \leq 1400 \\
0 & & \text { otherwise }
\end{array}\right.
$$

\begin{tabular}{|c|c|c|c|c|c|c|c|c|}
\hline \multirow{2}{*}{$\begin{array}{l}\text { Test } \\
\text { number }\end{array}$} & \multicolumn{2}{|c|}{$H_{0}(\mu)$} & \multicolumn{2}{|c|}{$H_{1}(\mu)$} & \multirow[t]{2}{*}{$p_{01}$} & \multirow[t]{2}{*}{$p_{10}$} & \multirow{2}{*}{$\begin{array}{l}\text { Accepted } \\
\text { hypothesis }\end{array}$} & \multirow[t]{2}{*}{$C F$} \\
\hline & $\mu-3$ & $3<\mu \leq 4$ & $\mu$ & $0<\mu \leq 1$ & & & & \\
\hline \multirow[t]{3}{*}{1} & $5-\mu$ & $4<\mu \leq 5$ & $2-\mu$ & $1<\mu \leq 2$ & 0.019 & 0.244 & $\tilde{H}_{1}$ & 0.927 \\
\hline & 0 & o.w. & 0 & o.w. & & & & \\
\hline & $\mu-3$ & $3<\mu \leq 4$ & $\mu+5$ & $-5<\mu \leq-4$ & & & & \\
\hline \multirow[t]{3}{*}{2} & $5-\mu$ & $4<\mu \leq 5$ & $-3-\mu$ & $-4<\mu \leq-3$ & 0.019 & $\simeq 0$ & $\tilde{H}_{0}$ & 0.99 \\
\hline & 0 & o.W. & 0 & o.w. & & & & \\
\hline & $\mu-3$ & $3<\mu \leq 4$ & $\int \frac{\mu+1}{2}$ & $-1<\mu \leq 1$ & & & & \\
\hline \multirow[t]{3}{*}{3} & $5-\mu$ & $4<\mu \leq 5$ & $\frac{3-\mu}{2}$ & $1<\mu \leq 3$ & 0.019 & 0.283 & $\tilde{H}_{1}$ & 0.937 \\
\hline & 0 & o.w. & 0 & o.w. & & & & \\
\hline & $\frac{\mu-2}{2}$ & $2<\mu \leq 4$ & $\frac{\mu+1}{2}$ & $-1<\mu \leq 1$ & & & & \\
\hline \multirow[t]{3}{*}{4} & $\frac{6-\mu}{2}$ & $4<\mu \leq 6$ & $\frac{3-\mu}{2}$ & $1<\mu \leq 3$ & 0.041 & 0.283 & $\tilde{H}_{1}$ & 0.873 \\
\hline & 0 & o.W. & 0 & o.w. & & & & \\
\hline & $\frac{\mu+4}{8}$ & $-4<\mu \leq 4$ & $\mu$ & $-1<\mu \leq 1$ & & & & \\
\hline \multirow[t]{2}{*}{5} & $5-\mu$ & $4<\mu \leq 5$ & $\frac{5-\mu}{4}$ & $1<\mu \leq 3$ & 0.527 & 0.452 & $\tilde{H}_{0}$ & 0.538 \\
\hline & 0 & o.w. & 0 & o.w. & & & & \\
\hline
\end{tabular}

Table 1 The results of five different tests in Example 4 


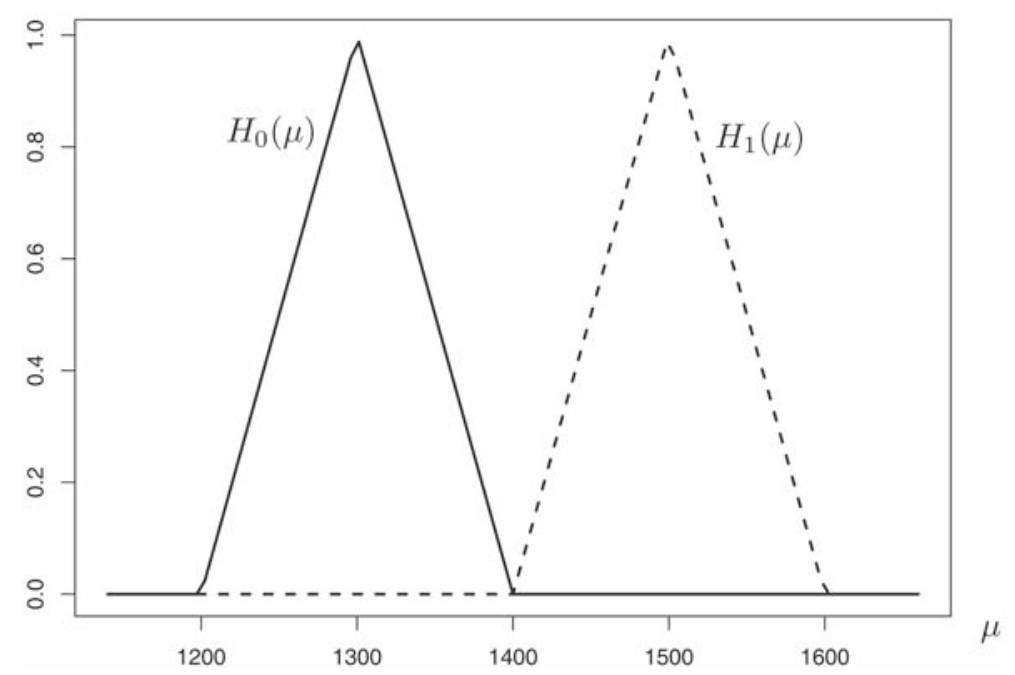

Fig. 5 The membership functions of fuzzy hypotheses in Example 5

and

$$
H_{1}(\mu)=\left\{\begin{array}{llr}
\frac{\mu-1400}{100} & \text { if } & 1400<\mu \leq 1500 \\
\frac{1600-\mu}{100} & \text { if } & 1500<\mu \leq 1600 \\
0 & & \text { otherwise }
\end{array}\right.
$$

Considering Definitions 3 and 4 , we have $H_{j b}(\mu)=H_{j}(\mu)$ and $H_{j b}^{*}(\mu)=\frac{H_{j b}(\mu)}{100}$, for $j=0,1$. In this example, fuzzy hypotheses can be modelled by form $(i i)$ and the rejection region for testing $\tilde{H}_{0}$ versus $\tilde{H}_{1}$ is of form (1.b). Therefore, considering Relation (3), one can compute $p$-value based on the weighted p.d.f. of $X$ under $\tilde{H}_{0 b}$ as follows:

$$
\begin{aligned}
p_{01} & =P_{\tilde{H}_{0 b}}(\bar{X} \geq 1327) \\
& =\int_{\mu} H_{0 b}^{*}(\mu) P_{\mu}(\bar{X} \geq 1327) d \mu \\
& =\int_{1200}^{1300} \frac{\mu-1200}{100^{2}}\left[1-\Phi\left(\frac{1327-\mu}{120 / \sqrt{36}}\right)\right] d \mu+\int_{1300}^{1400} \frac{1400-\mu}{100^{2}}\left[1-\Phi\left(\frac{1327-\mu}{120 / \sqrt{36}}\right)\right] d \mu \\
& =0.285 .
\end{aligned}
$$

Similarly, $p_{10}=P_{\tilde{H}_{1 b}}(\bar{X} \leq 1327)=\int_{\mu} H_{1 b}^{*}(\mu) P_{\mu}(\bar{X} \leq 1327) d \mu=2.95 \times 10^{-7}$. Therefore, $\tilde{H}_{0}$ is strongly accepted against $\tilde{H}_{1}$ with confidence factor $\mathrm{CF}=\frac{0.285}{0.285+2.95 \times 10^{-7}}=$ 0.999 .

Example 6. The manager of a factory has reinstalled a new system to upgrade the security of his personnel. We can suppose that the number of monthly accidents has a Poisson distribution with unknown mean $\lambda$. A study shows that 27 accidents have occurred during the past year. Having installed the new system, the manager wants to test if the average of the monthly accidents is approximately bigger than 3 . That is, to test

$$
\left\{\begin{array}{l}
\tilde{H}_{0}: \lambda \text { is approximately bigger than } 3, \\
\tilde{H}_{1}: \lambda \text { is approximately smaller than } 3
\end{array}\right.
$$

where their membership functions are 


$$
H_{0}(\lambda)=\left\{\begin{array}{llr}
1 & \text { if } & \lambda>3.25 \\
2(\lambda-2.75) & \text { if } & 2.75<\lambda \leq 3.25 \\
0 & \text { if } & \lambda \leq 2.75
\end{array}\right.
$$

and $H_{1}(\lambda)=1-H_{0}(\lambda)$ (see Fig. 6). Considering Definitions 3 and $4, H_{j b}^{*}(\lambda)=4 H_{j b}(\lambda)=$ $4 H_{j}(\lambda) I(2.75<\lambda \leq 3.25)$, where $j=0,1$. Fuzzy hypotheses are modelled by (iii) in this example, and the rejection region for testing $\tilde{H}_{0}$ versus $\tilde{H}_{1}$ is of form (1.a). By assumption, we have $T(\mathbf{X})=\sum_{i=1}^{12} X_{i} \sim \operatorname{Poisson}(12 \lambda)$ while $\mathbf{X}=\left(X_{1}, \ldots, X_{12}\right)$, and therefore one can compute $p_{01}$ and $p_{10}$ by Relations (2) and (3), respectively, as follows:

$$
\begin{array}{rl}
p_{01} & =P_{\tilde{H}_{0 b}}(T(\mathbf{X}) \leq 27) \\
& =\int_{\lambda} H_{0 b}^{*}(\lambda) P_{\lambda}\left(\sum_{i=1}^{12} X_{i} \leq 27\right) d \lambda \\
& =\int_{2.75}^{3.25} 8(\lambda-2.75) \sum_{t=0}^{27} \frac{e^{-12 \lambda}(12 \lambda)^{t}}{t !} d \lambda \\
& =0.059, \\
p_{10}=\int_{2.75}^{3.25} & 4[1-2(\lambda-2.75)]\left[1-\sum_{t=0}^{27} \frac{e^{-12 \lambda}(12 \lambda)^{t}}{t !}\right] d \lambda \\
=0.895 .
\end{array}
$$

Therefore, $p_{01}<p_{10}$, and $\tilde{H}_{1}$ is accepted against $\tilde{H}_{0}$ with confidence factor $\mathrm{CF}=$ $\frac{0.895}{0.059+0.895}=0.938$.

\section{Application to Agricultural Studies}

This applied example was conducted on an agriculturally polluted soil with $\mathrm{CdNO}_{3}$ salt in a laboratory at Tehran University, Iran [10]. Suppose that we are going to have an investigation on the amount of cadmium $(\mathrm{Cd})$ absorption in a plant from a polluted soil with $\mathrm{CdNO}_{3}$ salt. The unknown parameter is the amount of $\mathrm{Cd}$ absorption in a plant (in terms

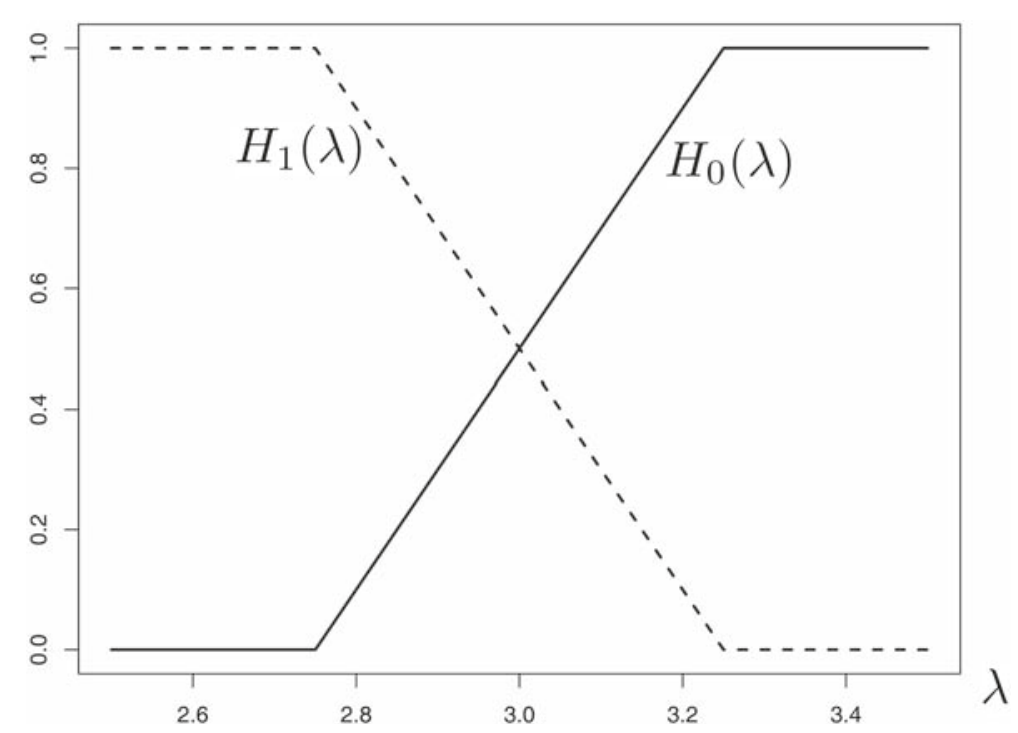

Fig. 6 The membership functions of fuzzy hypotheses in Example 6 
of mg. $\mathrm{kg}^{-1}$ dry matter) from soil which we denoted it with $\mu$. The optimum range of Cd absorbed in a plant has been proposed by Pais and Benton [12] as [0.05, 0.2], and also its maximum has been specified with $3 \mathrm{mg} \cdot \mathrm{kg}^{-1}$ dry matter. The experimenter wants to investigate on the following question: Whether the mean of $\mathrm{Cd}$ uptake coincides with the proposed suitable amounts by Pais and Benton or not?

In this applied example, one cannot represent the whole above presented information by Pais and Benton with a classical (precise) set. But, using fuzzy set theory, one can show the optimum range and the maximum amount of $\mathrm{Cd}$ uptakes in a plant by the following fuzzy set, in which the membership is considered to be one on the interval [0, 0.05], since the lower absorption of cadmium is better for any plant (Fig. 7)

$$
H_{0}(\mu)=\left\{\begin{array}{llr}
1 & \text { if } & 0 \leq \mu<0.2 \\
\frac{3-\mu}{2.8} & \text { if } & 0.2 \leq \mu<3 \\
0 & \text { if } & 3 \leq \mu
\end{array}\right.
$$

Now, the experimenter can test fuzzy hypotheses $\tilde{H}_{0}: \mu$ is $H_{0}(\mu)$, against $\tilde{H}_{1}: \mu$ is $H_{1}(\mu)$ without facing any contradiction in the result, where the membership functions of $\tilde{H}_{0}(\mu)$ and $\tilde{H}_{1}(\mu)=1-\tilde{H}_{0}(\mu)$ are shown in Fig. 7 . This is an advantage of considering fuzzy hypotheses rather than crisp hypotheses in some practical problems.

In order to test fuzzy hypothesis $\tilde{H}_{0}$ against $\tilde{H}_{1}$, the experimenter has recorded the following data for the amount of $\mathrm{Cd}$ absorption via below radish parts from a random sample of size $n=25$ pots: 1.33, 1.42, 1.56, 1.85, 1.89, 2.96, 2.97, 1.60, 2.02, 1.78, 1.98, $1.86,1.63,1.56,2.12,1.35,1.52,1.32,2.06,1.38,1.09,2.50,1.61,1.46$ and 2.18; see [13].

The sample mean is $\bar{x}=1.80 \mathrm{mg} \cdot \mathrm{kg}^{-1}$ dry matter and the sample standard deviation is $s=0.479 \mathrm{mg} \cdot \mathrm{kg}^{-1}$ dry matter. In this study, we assume that $X_{i} \sim N\left(\mu, s^{2}\right)$, for $i=1, \ldots, 25$, in which the unknown variance parameter can be estimated by a maximum likelihood estimator $s^{2}=\frac{1}{n} \sum_{i=1}^{n}\left(x_{i}-\bar{x}\right)^{2}$. Note that the normal distribution assumption for random variable $X_{i}$ comes from the essence of random variable $X_{i}$, which is rooted from nature. For instance, one can accept that the weight of seeds picked from a particular plant type, the absorption amount of heavy metals through the roots of a plant in a special greenhouse experiment, or the growth rate of plants in a specific time period, are all normal random variables with suitable means and variances.

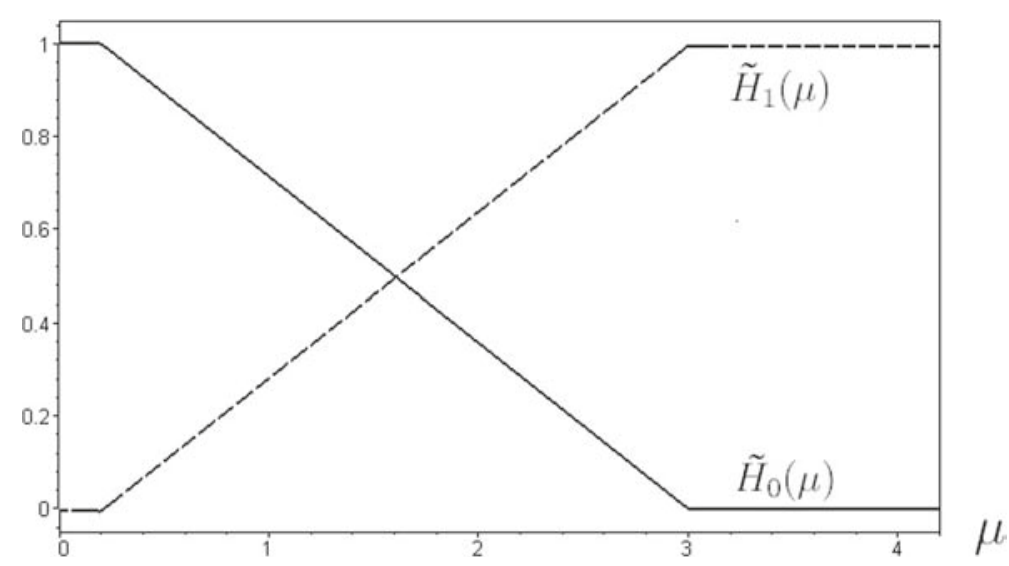

Fig. 7 The membership functions of the fuzzy hypotheses in agricultural example 


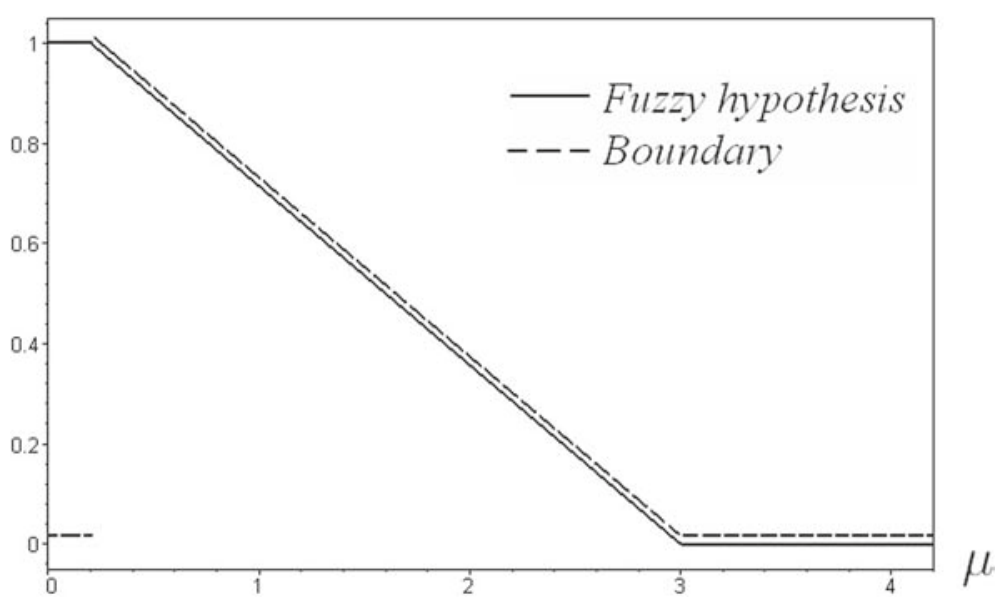

Fig. 8 The membership functions of the fuzzy null hypothesis and its boundary in agricultural example

Considering Definitions 3 and $4, H_{j b}^{*}(\mu)=\frac{H_{j b}(\mu)}{1.4}=\frac{H_{j}(\mu)}{1.4} I(0.2<\lambda \leq 3)$, where $j=0,1$. Also, the form of fuzzy hypothesis $\tilde{H}_{0}$ against $\tilde{H}_{1}$ is $(i v)$, hence the form of critical region is (1.b) and one can compute $p_{01}$ by Relation (3) as follows:

$$
\begin{aligned}
p_{01} & =P_{\tilde{H}_{0 b}}(\bar{X} \geq 1.80) \\
& =\int_{\mu} H_{0 b}^{*}(\mu) P_{\mu}(\bar{X} \geq 1.80) d \mu \\
& =\int_{0.2}^{3} \frac{(3-\mu) / 2.8}{1.4}\left[1-\Phi\left(\frac{1.80-\mu}{\frac{0.479}{\sqrt{25}}}\right)\right] d \mu \\
& =0.185 .
\end{aligned}
$$

Similarly, the form of fuzzy hypothesis $\tilde{H}_{1}$ against $\tilde{H}_{0}$ is (iii), hence the form of critical region is (1.a). So, one can compute $p_{01}$ by Relation (2) as follows:

$$
\begin{aligned}
p_{10} & =P_{\tilde{H}_{1 b}}(\bar{X} \leq 1.80) \\
& =\int_{\mu} H_{1 b}^{*}(\mu) P_{\mu}(\bar{X} \leq 1.80) d \mu \\
& =\int_{0.2}^{3} \frac{1-[(3-\mu) / 2.8]}{1.4} \Phi\left(\frac{1.80-\mu}{\frac{0.479}{5}}\right) d \mu \\
& =0.328 .
\end{aligned}
$$

Therefore, $p_{01}<p_{10}$, and so $\tilde{H}_{1}$ is accepted against $\tilde{H}_{0}$ with confidence factor $\mathrm{CF}=$ $\frac{0.328}{0.185+0.328}=0.639$. In other words, considering the confidence factor 0.639 , one can assert that the mean absorption $\mathrm{Cd}$ in the lower radish parts does not coincides with the proposed amounts by Pais and Benton [12] and so it is not suitable.

Note that if the experimenter decides to solve this problem by classical $p$-value method, first he/she must formulate the problem by one of the following hypotheses:

Test 1: $H_{0}: \mu \geq 0.2$ against $H_{1}: \mu<0.2$ and

Test 2: $H_{0}: \mu \geq 3$ against $H_{1}: \mu<3$,

while the results of tests 1 and 2 are in conflict with each other, for more details, see Tables $3-5$ of [13]. It must be noted that the presented contradiction in the result of tests 
1 and 2 comes from the difference between the null hypotheses in the two; in other words, it comes from very vague proposed information by Pais and Benton [12].

\section{Conclusions}

In this paper, a new $p$-value-based approach was presented for testing statistical hypotheses when the hypotheses are fuzzy rather than crisp. In contrast with the commonly $p$-value-based approach for testing fuzzy hypotheses, the decision rule in this approach is based on two $p$-values: (1) the $p$-value of testing fuzzy null hypothesis against fuzzy alternative hypothesis and (2) the $p$-value of testing fuzzy alternative hypothesis against fuzzy null hypothesis. On the basis of this idea, therefore, the introduced method has several advantages over the common methods. The main advantage is that the proposed method is based on both the null and alternative hypotheses. Several numerical examples and also an agricultural example were provided to illustrate the performance of the method. The study of testing fuzzy hypotheses in the framework of uncertainty theory is a potential topic for future work. Also, the study of testing fuzzy hypotheses using the introduced $p$ value and based on the paradigm of evidential statistics [16] is another potential topic for more research.

\footnotetext{
Author details

${ }^{1}$ Department of Statistics, Faculty of Mathematics and Computer, Shahid Bahonar University of Kerman, Kerman, Iran. ${ }^{2}$ Faculty of Engineering Science, College of Engineering, University of Tehran, Tehran, Iran. ${ }^{3}$ Department of Statistics, Faculty of Mathematical Science, Ferdowsi University of Mashhad, Mashhad, Iran.
}

Received: 27 August 2015 Accepted: 9 December 2015

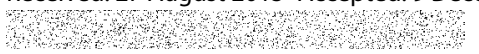

\section{References}

1. Arefi, M, Taheri, SM: Testing fuzzy hypotheses using fuzzy data based on fuzzy test statistic. J. Uncertain Sys. 5, 45-61 (2011)

2. Arefi, M, Taheri, SM: A new approach for testing fuzzy hypotheses based on fuzzy data. Int. J. Comput. Syst. $\mathbf{6}$, 318-327 (2013)

3. Arnold, BF: An approach to fuzzy hypothesis testing. Metrika. 44, 119-126 (1996)

4. Chachi, J, Taheri, SM, Viertl, R: Testing statistical hypotheses based on fuzzy confidence intervals. Austrian. J. Stat. 41, 267-286 (2012)

5. Emadi, M, Arghami, N: Some measures of support for statistical hypotheses. J. Stat. Theory and Appl. 2, 165-176 (2003)

6. Denœ ux, T, Masson, MH, Hébert, PA: Nonparametric rank-based statistics and significance tests for fuzzy data. Fuzzy Sets and Syst. 153, 1-28 (2005)

7. Fazlalipor Miyandoab, M, Arzideh, K, Farbod, D: Fuzzy hypothesis testing with fuzzy data by using fuzzy $p$-value. Acta Univ. Apulensis. 32, 293-307 (2012)

8. Filzmoser, P, Viertl, R: Testing hypotheses with fuzzy data: the fuzzy p-value. Metrika. 59, 21-29 (2004)

9. Geyer, C, Meeden, G: Fuzzy confidence intervals and P-values. Stat. Sci. 20, 358-387 (2005)

10. Ivani, R: The effect of various sources and amounts of organic fertilizer on bioavailability Zn and Cd in soil, M.Sc. Thesis, Faculty of Water and Soil. University of Tehran, Iran (2007)

11. Knight, K: Mathematical Statistics. Chapman \& Hall/CRC, Boca Raton (2000)

12. Pais, I, Benton, JJ: The Handbook of Trace Elements. St. Lucie Press, Florida (1997)

13. Parchami, A, Ivani, R, Mashinchi, M: An application of testing fuzzy hypotheses: a soil study on bioavailability of Cd. Scientia Iranica. 18,470-478 (2011)

14. Parchami, A, Taheri, SM, Mashinchi, M: Fuzzy p-value in testing fuzzy hypotheses with crisp data. Stat. Papers. 51 209-226 (2010)

15. Parchami, A, Taheri, SM, Mashinchi, M: Testing fuzzy hypotheses based on vague observations: a $p$-value approach Stat. Papers. 53, 469-484 (2012)

16. Royall, R: Statistical Evidence: A Likelihood Paradigm. Chapman and Hall/CRC, Florida (1997)

17. Royall, R: On probability of observing misleading statistical evidence. J. Am. Stat. Assoc. 95, 760-768 (2000)

18. Taheri, SM, Behboodian, J: Neyman-Pearson Lemma for fuzzy hypotheses testing. Metrika. 49, 3-17 (1999)

19. Tanaka, H, Okuda, T, Asai, K: Fuzzy information and decision in a statistical model. In: Gupta, MM, et al (eds.) Advances in Fuzzy Set Theory and Applications, pp. 303-320. Amsterdam, North-Holland, (1979)

20. Torabi, H, Behboodian, J: Sequential probability ratio test for fuzzy hypotheses testing with vague data. Austrian J. Statistics. 34, 25-38 (2005)

21. Viertl, R: Univariate statistical analysis with fuzzy data. Comput. Stat. Data Anal. 51, 133-147 (2006)

22. Zadeh, LA: Probability measures of fuzzy events. J. Math. Anal. Appl. 23, 421-427 (1968)

23. Zadeh, LA: Fuzzy sets. Inf. Control. 8, 338-359 (1965) 